\title{
Research Progress of Mechanism of Action of Resveratrol
}

\author{
Wenzhe Dong, Yan Zhou, Zhifang Yang* \\ Shanghai University of Medicine and Health Sciences, Shanghai, China \\ Email: "guicaitang@163.com
}

Received 7 March 2016; accepted 23 April 2016; published 26 April 2016

Copyright (C) 2016 by authors and Scientific Research Publishing Inc.

This work is licensed under the Creative Commons Attribution International License (CC BY).

http://creativecommons.org/licenses/by/4.0/

(c) (i) Open Access

\begin{abstract}
Resveratrol is a kind of polyphenolic compound that widely exists in plants and has extensive physiological pharmacological function and is very useful for human health. For the mechanism of action and function of $\mathrm{RV}$, people are doing a variety of groundbreaking researches. Results show that the molecular mechanism of RV is embodied in aspects such as oxidative stress and diseases of neuropathy, anti-oxygenation and pro-oxidant effect and RV target molecule, etc. This article mainly summarized the mechanism of action of resveratrol in these aspects.
\end{abstract}

\section{Keywords}

Resveratrol, Oxidative Stress, Antioxidant Effect, Pro-Oxidant Effect, Target Molecule of Resveratrol

\section{Introduction}

Resveratrol (3,5,4'trihydroxy-trans-toluylene, Resveratrol, RV) is a polyphenolic plant antitoxin and exists in a variety of plant species. It is widely known that it exists in red wine. It is verified in animal experiments that RV has beneficial effects on human health. RV shows significant biological activity, including antineoplastic activity, antitubulin activity and cardiovascular disease resistance activity, etc. A number of studies have shown that $\mathrm{RV}$ stimulates the production of endothelial nitric oxide, reduces oxidative stress, restrains vascular inflammation and prevents platelet aggregation. Besides, a series of RV derivatives have been compounded through entering into other functional groups to increase the valence or enhance the activity of specific properties of RV. There are many pioneering researches on RV. One of the researches shows that RV inhibits the metabolism of arachidonic acid in granular cells through combined action with 5-lipoxygenase and cyclooxygenase [1]. In reality, before 1992, there were very few people who were interested in RV until it is supposed to be the factor in red wine to protect the heart. After that, many researches on RV are carried out. People find it has many func*Corresponding author. 
tions, such as slowing down cancer progression, relieving ischemic injury and enhancing anti-stress force as well as prolonging life-span, etc. However, there are many limitations of this drug. The most important one is its slow oral bioavailability, due to rapid excretion and extensive metabolism into variants of glucuronide and sulfonated conjugates [2]. At the same time, we should remember that the drug is not much soluble in water [3]. This article looks forward to reviewing and summarizing molecular mechanism related to RV.

\section{Oxidative Stress and Neurodegenerative Diseases}

Free radicals with proper numbers are necessary for life entity, because they involve in the signal transduction of cells and phagocytosis of phagocyte [4]. However, the aging process and exact degenerative disease have important relationships with unnecessary products of reactive oxygen species (ROS). Therefore, for human health, the metabolism or free radical produced from environmental resources and antioxidant defense system such as superoxide dismutase (SOD), catalase (CAT), glutathione peroxidase (GPx) are very important to rapidly eliminate and neutralize the balance between free radicals.

In pathology related to oxidative stress, the neurodegenerative diseases occupy explicit direction, because nerve cells are easy to be attacked by free radicals. Oxidative stress is the main pathogenesis for various lateonset diseases. Nervous system includes brain, spinal cord and peripheral nervous. Its high vulnerability for oxidative stress is because these organizations have extremely high biological energy and quantity demanded for oxygen. In reality, the neuron axon and multiple synapses have high demands for ATP. In the process of breathing, they are mainly responsible for enormous oxygen expenditure of brain; in addition, high contents of lipid in multiple regions of the brain and iron that are easy to be mobilized can stimulate the produce of ROS. Because the reduction of cranial capacity due to age can offset oxidative stress as well as lead to irreversible damage, it helps to research on the pathogenesis of neurodegenerative diseases. At the level of pathology, almost all the neurodegenerative diseases have common characteristics, such as the produce of misfolded protein aggregates, the abnormality of metal ion and oxidative stress [5]. Generally speaking, protein aggregates are mainly composed of typical proteins of different diseases. For example, in Alzheimer's disease(AD), the extracellular senile plaques are composed of $\beta$-amyloid protein $(\mathrm{A} \beta)$, and it comes from amyloid precursor protein (APP) because of the mutation of gene coding; intracellular tangles come from tau protein; Huntington's.

Disease(HD) is because of gene mutation, and then influences the structure and aggregation tendency of Huntingdon protein (htt); [6] in Parkinson's disease (PD), the Louis corpuscles aggregated in cytoplasm are mainly composed of $\alpha$-synuclein and ubiquitin; [7] related genes of different series have similar protein products, including SOD1, TDP43, FUS,UBQLN2, and C9OKF72. They are also found in neuronal aggregates in Amyotrophic lateral sclerosis (ALS) patients [8].

Besides, the change of iron is tested in disseminated sclerosis, spastic paraplegia and ALS. It is believed that the accumulation of iron and related neural degeneration are secondary changes. This may be due to the change of blood brain barrier led by the form or inflammation of abnormal blood vessels of the organizations [9].

Practice has proved that in $\mathrm{AD}$, markers of oxidative stress appear before pathological lesion, including causing age pigment and neurofibrillary tangles. In addition, the exhaustion of ATP induced by ROS or peroxidatic reaction between lipid and protein has close relationships with Parkinsonism and the process of neuron necrosis; [10] the protein oxidative damage appears in the form of increasing of protein carbonyl and 8-Hydroxy2'-deoxyguanosine. This kind of change exists in PD brain. Some evidence suggests that the nitration and nitrosylation of particular proteins are because of active nitrogen [11]. Under the circumstances, a large number of studies have shown that neurodegenerative diseases have relationships with oxidative stress. More and more experimental results show that antioxidant and scavenger substances play a clear role in preventing nervous tissue from being damaged by oxidative stress. It is obvious that restricting the produce of free radical, oxidative stress and damage can slow down the development process of neurodegenerative diseases. In reality, researchers have great interest in learning to obtain natural-occurring substances that can protect neuron from plants. There are some kinds of native compound that has anti-oxygenation can withstand oxidative stress through eliminating excess free radicals to prevent them from having a series of reactions with diseases. Under such circumstances, because of its chemical properties, RV is a very promising compound to withstand neuropathy.

\section{Anti-Oxygenation and Pro-Oxidant Effect of RV}

The anti-oxygenation and pro-oxidant effect of RV are different because of different dosage or cell types. In 
particular, with the increase of drug concentration, RV has stronger anti-oxygenation. The research conducted by Cavallaro [12] and others shows that no matter in low concentration or high concentration, RV can inhibit the produce of superoxide anion.

RV transforms hydrogen atom or electron into free radicals and then plays the role of resisting oxidation and eliminating free radicals [13]. Under the circumstances, the special position of hydroxyl groups plays a key role. Among them, the 4'-hydroxide radical has the strongest activity [14]. The chemical properties of RV make it have anti-oxygenation: in reality, this kind of drug molecules has conjugated double bond formed by two phenolic groups to make the electron more discrete. The research on oxy-resveratrol shows that the drug after modification has stronger antioxidant activity. It may be because that additional hydroxyl makes oxy-RV have better hydrogen donor [15]. Therefore, the likely antioxidation mechanism is that RV provides hydrogen ion to free radicals, and then restrains peroxidation and protects DNA molecule, lipoid and protein.

Recently, using the planar lipid bilayer and liposome model, with low dosage, RV acts on polar groups on the surface; with high dosage, RV distributes outside the lipid bilayer. It is interesting that the distribution of RV has close relationship with its anti-oxygenation, because the polyphenol located in lipid bilayer can effectively prevent the lipid peroxidation [16]. The RV inside red blood cells reacts with protoheme, which can protect the protein from the damage of peroxidation [17]. The RV constitutes phenoxy radicals through eliminating free radicals and forms stable resonance, and then destroys lipid peroxidation chain.

RV has certain anti-oxygenation for 1,1-diphenyl-2-trinitrobenzene hydrazine (DPPH) free radicals, and then significantly reduces superoxide anion, inhibits the oxidation of protoheme, and finally reduces the concentration of superoxide [17] [18]. The reason why the protoheme of RV has anti-oxygenation is that its phenol groups change $\mathrm{Fe}^{3+}$ into $\mathrm{Fe}^{2+}$. It is well-known that $\mathrm{RV}$ can inhibit the oxidation reaction of low density lipoprotein (LDL) and effectively inhibit the form of atherosclerotic plaque [19]. In the process of preventing catalytic oxidation of copper, RV is more effective than progesterone. Besides, RV can effectively maintain the activity of some antioxidase, such as glutathione peroxidase (GPx), glutathione-s-transferase (GST) and superoxide dismutase (SOD) [20]. It is mentioned in many researches that the reason why RV has many beneficial effects is that it can inhibit the composition of inducible nitric oxide synthase (Inos) and increase the nitric oxide synthase (eNOS) in blood vessel endothelium [21]. These may reveal why RV has beneficial effects on Alzheimer disease, and the molecular mechanism.

On the other hand, the research results of Ahmad show that when superoxide anion in the endochylema increases and cupric ion exists, RV with low dosage shows pro-oxidant effects [22]. To be specific, the combination of RV and cupric ion can promote the pro-oxidant effects of drugs [23].

\section{RV Target Molecule}

Although for the function of RV, everyone pays attention to its anti-oxygenation. There are still some researches to show that this drug has very broad effects on signal transduction pathway.

Some researches based on experiments in vitro and in vivo show that RV can regulate the activities of a variety of living things. And these activities have relationships with growth and differentiation of cells and apoptosis, angiogenesis and metabolism [24].

Therefore, RV can terminate the carcinogenic process through many signal pathways and prolong one or more stages in the process. Meanwhile, it is verified that RV can inhibit excessive enzyme of different kinds, including but not limited to kinase, esterase and cyclooxygenase, etc. Besides, some researches show that RV can induce the termination of cell cycle of many tumor cell lines through modulating gap-associated protein of cyclin-dependent kinases (CDK) or through influencing the activity of dependent or independent pathway of tumor suppressor proteins P53 [25]. P53 has relations with cell proliferation and apoptosis, so it plays a key role in preventing the occurrence of cancer.

Besides, RV can regulate the effect of SIRT-1. SIRT-1 is a highly conserved protein family and has many potential therapeutic targets of human diseases, such as diabetes, inflammatory diseases and neuropathic degenerative diseases [26].

RV rivals the rising calcium and neurovirulence produced by ASL and has anti-oxygenation. In the experiment of cell culture, it is verified that RV can withstand glutamate toxicity and then protect neuron, and can reduce the expression of glycogen synthase kinase-3 (GSK-3 $\beta$ ) through P13K/Akt pathway [27]. GSK-3 $\beta$ has relationship with many signal pathways. Most of it exists in endochylema, and small amounts of it exist in cell 
nucleus or mitochondria. It can adjust the pathways of cell death under stress state. A series of researches on cerebral blood flow (CBF) and cognitive competence of human show that RV can adjust brain function and improve sugar metabolism as well as promote the angiectasis through increasing the compound of endothelial nitric oxide synthase or nitric oxide [28].

$\mathrm{RV}$ can promote the length of telomere through adjusting ATP dependent DnaB called Werner syndrome; this kind of protective effect has important significance on mitochondrial function and oxidative stress, because the shortening of telomere length is the same as activation of P53. Both of them can inhibit the damages for peroxidase proliferation activated receptor $\gamma$ co-activator $1 \alpha(\mathrm{PGC}-1 \alpha)$ and mitochondria [29]. RV can activate PGC$1 \alpha$ deacetylation through acting on SIRT1. PGC- $1 \alpha$ can induce the transcription of nuclear respiratory factors 1 and 2 (NRF1, NRF2), and then realize the increase of transcription of mitochondrial respiratory factor (mtTFA) and nuclear-encoded mitochondrial subunit [30] of other compound of electron transport chain, and finally effectively increase the mitochondrial respiration and biological function.

In research on cell model of Parkinson's disease, RV activates SIRT1 pathway of AMP protein kinase, increases inhibitor of apoptosis protein (IAP) Bcl-2 and reduces the expression of Bax protein; and enhances antioxidant sulfide reduction protein 2 (TRX2) and anti-apoptosis protein related to X chromosome and plays the role of antioxidant of mitochondria [31]. Another study shows that RV can increase the expression of Bcl-2 so that it can prevent the apoptosis of neuron [32]. RV can effectively relieve inflammatory reaction. This drug can weaken the activity of inflammatory cells, reduce inflammatory complication and inhibit transcription factor as well as reduce the release of inflammatory medium [33].

\section{Conclusion}

Resveratrol has enormous potential and development prospect to treat various diseases. Many experiments show that the effective dose of resveratrol in vivo is at least above three times of effective dose in vitro. And it has relatively good security. This may be the effects that resveratrol has through many ways. We believe that resveratrol is beneficial to human health, because it can delay senescence and relieve sub-health caused by unhealthy life styles. The data of experiments in vivo and in vitro of animals increase the possibility of clinical test on human. But at present, there is no powerful evidence to prove that it is safe to apply RV to human chronically. In the future, the research on resveratrol should pay attention to dose-effect relationship of human, bioavailability, valence and direct and indirect mechanism of action, looking forward to provide guidance for clinical test.

\section{Acknowledgements}

This manuscript was supported by Shanghai Science and Technology Development Funds (Fund No. B230214001) and Shanghai Vocational Connotation Construction Funds (Fund No. FA1-3903-16-117066).

\section{References}

[1] Kimura, Y., Okuda, H. and Arichi, S. (1985) Effects of Stibenes on Arachidonate Metabolism in Leukocytes. Biochimica et Biophysica Acta, 834, 275-278.

[2] Walle, T., Hsieh, F., DeLegge, M.H., Oatis Jr., J.E. and Walle, U.K. (2004) High Absorption But Very Low Bioavailability of Oral Resveratrol in Humans. Drug Metabolism and Disposition, 32, 1377-1382. http://dx.doi.org/10.1124/dmd.104.000885

[3] Walle, T. (2011) Bioavailability of Resveratrol. Annals of the New York Academy of Sciences, 1215, 9-15. http://dx.doi.org/10.1111/j.1749-6632.2010.05842.x

[4] Forman, H.J. (2010) Reactive Oxygen Species and Alpha, Beta-Unsaturated Aldehydes as Second Messengers in Signal Transduction. Annals of the New York Academy of Sciences, 1203, 35-44. http://dx.doi.org/10.1111/j.1749-6632.2010.05551.x

[5] Granzotto, A. and Zatta, P. (2014) Resveratrol and Alzheimer's Disease: Message in a Bottle on Red Wine and Cognition. Frontiers in Aging Neuroscience, 6, 95. http://dx.doi.org/10.3389/fnagi.2014.00095

[6] La Spada, A.R. (2012) Finding a Sirtuin Truth in Huntington's Disease. Nature Medicine, 18, 24-26. http://dx.doi.org/10.1038/nm.2624

[7] Blesa, J., Phani, S., Jackson-Lewis, V., et al. (2012) Classic and New Animal Models of Parkinson's Disease. Journal of Biomedicine and Biotechnology, 2012, Article ID: 845618. http://dx.doi.org/10.1155/2012/845618 
[8] Herskovits, A.Z. and Guarente, L. (2013) Sirtuin Deacetylases in Neurodegenerative Diseases of Aging. Cell Research, 23, 746-758. http://dx.doi.org/10.1038/cr.2013.70

[9] Gutteridge, J.M. (1986) Iron Promoters of the Fenton Reaction and Lipid Peroxidation Can Be Released from Haemoglobin by Peroxides. FEBS Letters, 201, 291-295. http://dx.doi.org/10.1016/0014-5793(86)80626-3

[10] Tatton, W.G., Chalmers-Redman, R., Brown, D., et al. (2003) Apoptosis in Parkinson's Disease: Signals for Neuronal Degradation. Annals of Neurology, 53, S61-S72.

[11] Seet, R.C., Lee, C.Y., Lim, E.C., Tan, J.J.H., Quek, A.M.L., Chong, W.-L., et al. (2010) Oxidative Damage in Parkinson Disease: Measurement Using Accurate Biomarkers. Free Radical Biology \& Medicine, 48, 560-566. http://dx.doi.org/10.1016/j.freeradbiomed.2009.11.026

[12] Cavallaro, A., Ainis, T., Bottari, C. and Fimiani, V. (2003) Effect of Resveratrol on Some Activities of Isolated and in Whole Blood Human Neutrophils. Physiological Research, 52, 555-562.

[13] Iuqa, C., Alvarez-Idaboy, J.R. and Russo, N. (2012) Antioxidant Activity of Trans-Resveratrol toward Hydroxyl and Hydroperoxyl Radicals: A Quantum Chemical and Computational Kinetics Study. The Journal of Organic Chemistry, 77, 3868-3877. http://dx.doi.org/10.1021/jo3002134

[14] Stivala, L.A., Savio, M., Carafoli, F., Perucca, P., Bianchi, L., Maga, G., et al. (2001) Specific Structural Determinants Are Responsible for the Antioxidant Activity and the Cell Cycle Effects of Resveratrol. The Journal of Biological Chemistry, 276, 22586-22594. http://dx.doi.org/10.1074/jbc.M101846200

[15] Chao, J., Yu, M.S., Ho, Y.S., Wang, M. and Chang, R.C.-C. (2008) Dietary Oxyresveratrol Prevents Parkinsonian Mimetic 6-Hydroxydopamine Neurotoxicity. Free Radical Biology \& Medicine, 45, 1019-1026. http://dx.doi.org/10.1016/j.freeradbiomed.2008.07.002

[16] Selvarai, S., Mohan, A., Narayanan, S., Sethuraman, S. and Krishnan, U.M. (2013) Dose-Dependent Interaction of Trans-Resveratrol with Biomembranes: Effects on Antioxidant Property. Journal of Medicinal Chemistry, 56, $970-981$. http://dx.doi.org/10.1021/jm3014579

[17] Tellone, E., De Rosa, M.C., Pirolli, D., Russo, A., Giardina, B., Galtieri, A. and Ficarra, S. (2014) Molecular Interactions of Hemoglobin with Resveratrol: Potential Protective Antioxidant Role and Metabolic Adaptations of the Erythrocyte. Biological Chemistry, 395, 347-354. http://dx.doi.org/10.1515/hsz-2013-0257

[18] Galtieri, A., Tellone, E., Ficarra, S., Russo, A., Bellocco, E., Barreca, D., et al. (2010) Resveratrol Treatment Induces Redox Stress in Red Blood Cells: A Possible Role of Caspase 3 in Metabolism and Anion Transport. Biological Chemistry, 391, 1057-1065. http://dx.doi.org/10.1515/bc.2010.100

[19] Pervaiz, S. (2003) Resveratrol: From Grapevines to Mammalian Biology. The FASEB Journal, 17, 1975-1985. http://dx.doi.org/10.1096/fj.03-0168rev

[20] de la Lastra, C.A. and Villegas, I. (2007) Resveratrol as an Antioxidant and Pro-Oxidant Agent: Mechanisms and Clinical Implications. Biochemical Society Transactions, 35, 1156-1160. http://dx.doi.org/10.1042/BST0351156

[21] Tsai, S.K., Hung, L.M., Fu, Y.T., Cheng, H., Nien, M.-W., Liu, H.-Y., Zhang, F.B.-Y. and Huang, S.-S. (2007) Resveratrol Neuroprotective Effects during Focal Cerebral Ischemia Injury via Nitric Oxide Mechanism in Rats. Journal of Vascular Surgery, 46, 346-353. http://dx.doi.org/10.1016/j.jvs.2007.04.044

[22] Ahmad, A., Syed, F.A., Singh, S. and Hadi, S.M. (2005) Prooxidant Activity of Resveratrol in the Presence of Copper Ions: Mutagenicity in Plasmid DNA. Toxicology Letters, 159, 1-12. http://dx.doi.org/10.1016/j.toxlet.2005.04.001

[23] Muqbil, I., Beck, F.W., Bao, B., Sarkar, F.H., Mohammad, R.M., Hadi, S.M. and Azmi, A.S. (2012) Old Wine in a New Bottle: The Warburg Effect and Anticancer Mechanisms of Resveratrol. Current Pharmaceutical Design, 18, 1645-1654. http://dx.doi.org/10.2174/138161212799958567

[24] Kundu, J.K. and Surh, Y.J. (2008) Cancer Chemopreventive and Therapeutic Potential of Resveratrol: Mechanistic Perspectives. Cancer Letters, 269, 243-261. http://dx.doi.org/10.1016/j.canlet.2008.03.057

[25] Fan, E., Jiang, S., Zhang, L. and Bai, Y. (2008) Molecular Mechanism of Apoptosis Induction by Resveratrol, a Natural Cancer Chemopreventive Agent. International Journal for Vitamin and Nutrition Research, 78, 3-8. http://dx.doi.org/10.1024/0300-9831.78.1.3

[26] Herskovits, A.Z. and Guarente, L. (2013) Sirtuin Deacetylases in Neurodegenerative Diseases of Aging. Cell Research, 23, 746-758. http://dx.doi.org/10.1038/cr.2013.70

[27] Simao, F., Matte, A., Pagnussat, A.S., Netto, C.A. and Salbego, C.G. (2012) Resveratrol Prevents CA1 Neurons against Ischemic Injury by Parallel Modulation of both GSK-3 $\beta$ and CREB through PI3-K/Akt Pathways. European Journal of Neuroscience, 36, 2899-2905. http://dx.doi.org/10.1111/j.1460-9568.2012.08229.x

[28] Kennedy, D.O., Wightman, E.L., Reay, J.L., Lietz, G., Okello, E.J., Wilde, A. and Haskell, C.F. (2010) Effects of Resveratrol on Cerebral Blood Flow Variables and Cognitive Performance in Humans: A Double-Blind, Placebo-Controlled, Crossover Investigation. The American Journal of Clinical Nutrition, 91, 1590-1597. 
http://dx.doi.org/10.3945/ajen.2009.28641

[29] Sahin, E., Colla, S., Liesa, M., Moslehi, J., Müller, F.L., Guo, M., et al. (2011) Telomere Dysfunction Induces Metabolic and Mitochondrial Compromise. Nature, 470, 359-365. http://dx.doi.org/10.1038/nature09787

[30] Scarpulla, R.C. (2011) Metabolic Control of Mitochondrial Biogenesis through the PGC-1 Family Regulatory Network. Biochimica et Biophysica Acta, 1813, 1269-1278. http://dx.doi.org/10.1016/j.bbamcr.2010.09.019

[31] Kairisalo, M., Bonomo, A., Hyrskyluoto, A., Mudò, G., Belluardo, N., Korhonen, L. and Lindholm, D. (2011) Resveratrol Reduces Oxidative Stress and Cell Death and Increases Mitochondrial Antioxidants and XIAP in PC6.3-Cells. Neuroscience Letters, 488, 263-266. http://dx.doi.org/10.1016/j.neulet.2010.11.042

[32] Jin, F., Wu, Q., Lu, Y.F., Gong, Q.-H. and Shi, J.-S. (2008) Neuroprotective Effect of Resveratrol on 6-OHDA-Induced Parkinson's Disease in Rats. European Journal of Pharmacology, 600, 78-82. http://dx.doi.org/10.1016/j.ejphar.2008.10.005

[33] Zhang, F., Shi, J.S., Zhou, H., Wilson, B., Hong, J.-S. and Gao, H.-M. (2010) Resveratrol Protects Dopamine Neurons against Lipopolysaccharide-Induced Neurotoxicity through Its Anti-Inflammatory Actions. Molecular Pharmacology, 78, 466-477. http://dx.doi.org/10.1124/mol.110.064535 